\title{
Four-Neutrino Mass Spectra and the Super-Kamiokande Atmospheric Up-Down Asymmetry
}

\author{
S.M. Bilenky \\ Joint Institute for Nuclear Research, Dubna, Russia, and \\ Institute for Theoretical Physics, University of Vienna, \\ Boltzmanngasse 5, A-1090 Vienna, Austria \\ C. Giunti \\ INFN, Sezione di Torino, and Dipartimento di Fisica Teorica, Università di Torino, \\ Via P. Giuria 1, I-10125 Torino, Italy \\ W. Grimus and T. Schwetz \\ Institute for Theoretical Physics, University of Vienna, \\ Boltzmanngasse 5, A-1090 Vienna, Austria
}

(22 March 1999)

\begin{abstract}
In the framework of schemes with mixing of four massive neutrinos, which can accommodate atmospheric, solar and LSND ranges of $\Delta m^{2}$, we show that, in the whole region of $\Delta m_{\mathrm{LSND}}^{2}$ allowed by LSND, the Super-Kamiokande up-down asymmetry excludes all mass spectra with a group of three close neutrino masses separated from the fourth mass by the LSND gap of order $1 \mathrm{eV}$. Only two schemes with mass spectra in which two pairs of close masses are separated by the LSND gap can describe the Super-Kamiokande up-down asymmetry and all other existing neutrino oscillation data.
\end{abstract}

UWThPh-1999-20, DFTT 17/99, hep-ph/9903454

The observation of a significant up-down asymmetry of atmospheric high-energy $\stackrel{(-)}{\nu}$-induced events in the Super-Kamiokande experiment [1] is considered as the first model-independent evidence in favor of neutrino oscillations. Such indications were also obtained in other atmospheric neutrino experiments: Kamiokande [2], IMB [3], Soudan-2 [4] and MACRO [5]. In addition, evidence in favor of neutrino masses and mixing is provided by all solar neutrino experiments: Homestake [6], Kamiokande [7], GALLEX [8], SAGE [9] and Super-Kamiokande [10]. Finally, observation of $\bar{\nu}_{\mu} \rightarrow \bar{\nu}_{e}$ and $\nu_{\mu} \rightarrow \nu_{e}$ oscillations have been claimed by the LSND collaboration [11]. For the explanation of all these data three different scales of neutrino mass-squared differences are required: $\Delta m_{\mathrm{sun}}^{2} \sim$ $10^{-10} \mathrm{eV}^{2}$ (vacuum oscillations) or $\Delta m_{\text {sun }}^{2} \sim 10^{-5} \mathrm{eV}^{2}$ (MSW), $\Delta m_{\mathrm{atm}}^{2} \sim 10^{-3} \mathrm{eV}^{2}, \Delta m_{\mathrm{LSND}}^{2} \sim 1 \mathrm{eV}^{2}$. Thus, at least four neutrinos with definite mass are needed to describe all data.

Four-neutrino schemes have been considered in many papers. For early works see Ref. [12] and for a more comprehensive list of four-neutrino papers consult, e.g., Ref. [13]. In Refs. [14 16] it was shown that from the results of all existing experiments, including short-baseline (SBL) reactor and accelerator experiments in which no indications of neutrino oscillations have been found, information on the four-neutrino mass spectrum can be inferred. In the case of three different scales of $\Delta m^{2}$, there are two different classes of neutrino mass spectra (see Fig. 1) that satisfy the inequalities $\Delta m_{\text {sun }}^{2} \ll$ $\Delta m_{\mathrm{atm}}^{2} \ll \Delta m_{\mathrm{LSND}}^{2}$. In the spectra of class 1 there is a group of three close masses which is separated from the fourth mass by the LSND gap of around $1 \mathrm{eV}$. It contains the spectra (I) - (IV) in Fig. 11. Note that spectrum (I) corresponds to a mass hierarchy, spectrum (III) to an inverted mass hierarchy, whereas (II) and (IV) are non-hierarchical spectra. In the spectra of class 2 there are two pairs of close masses which are separated by the LSND gap. The two possible spectra in this class are denoted by (A) and (B) in Fig. 1.

It was shown in Ref. 114 that, in the case of the spectra of class 1, from the existing data one can obtain constraints on the amplitude of SBL $\nu_{\mu} \rightarrow \nu_{e}$ oscillations that are not compatible with the results of the LSND experiment in the allowed region $0.2 \mathrm{eV}^{2} \lesssim \Delta m_{\text {LSND }}^{2} \lesssim$ $2 \mathrm{eV}^{2}$ with the exception of the small interval from 0.2 to $0.3 \mathrm{eV}^{2}$. In Ref. 114 the double ratio $R$ of $\mu$-like over $e$-like events has been used as input from atmospheric neutrino measurements, whereas in the present letter we consider what constraints on neutrino mixing can be inferred from the up-down asymmetry of multi-GeV muonlike events measured in the Super-Kamiokande experiment [17], i.e., from

$$
A_{\mu}=\frac{U-D}{U+D}=-0.311 \pm 0.043 \pm 0.01,
$$

where $U$ and $D$ denote the number of events in the zenith angle ranges $-1<\cos \theta<-0.2$ and $0.2<\cos \theta<1$, respectively. We will show that with this input the conclusion of Ref. 14 will be strengthened and that now the neutrino mass spectra of class 1 are disfavored for any value of $\Delta m_{\mathrm{LSND}}^{2}$ in the allowed range. In addition, we will also derive a constraint on the mixing matrix for the neutrino mass spectra (A) and (B). 
$千 m_{4}$

(I)
(II)

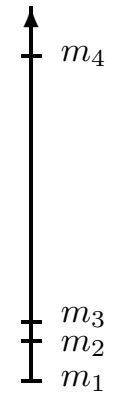

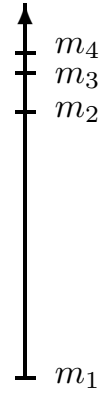

(III)

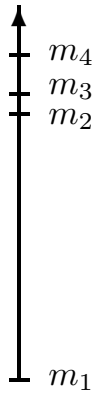

(IV)

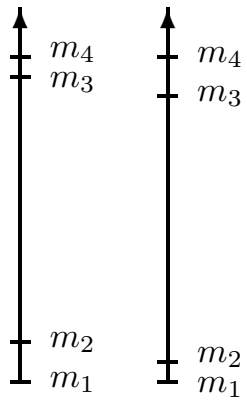

(A)
(B)
FIG. 1. The six types of neutrino mass spectra that can accommodate the solar, atmospheric and LSND scales of $\Delta m^{2}$. The different distances between the masses on the vertical axes symbolize the different scales of $\Delta m^{2}$. The spectra (I) - (IV) define class 1 , whereas class 2 comprises (A) and (B).

The general case of mixing of four massive neutrinos is described by $\nu_{\alpha L}=\sum_{j=1}^{4} U_{\alpha j} \nu_{j L}$, where $U$ is the $4 \times 4$ unitary mixing matrix, $\alpha=e, \mu, \tau, s$ denotes the three active neutrino flavors and the sterile neutrino, respectively, and $j=1, \ldots, 4$ enumerates the neutrino mass eigenfields. For definiteness, we will consider the spectrum of type I with a neutrino mass hierarchy $m_{1} \ll m_{2} \ll m_{3} \ll m_{4}$, but the results that we will obtain in this case will apply to all spectra of class 1 .

The probability of SBL $\nu_{\mu} \rightarrow \nu_{e}$ transitions is given by the two-neutrino-like formula 14

$$
P_{\nu_{\mu} \rightarrow \nu_{e}}=P_{\bar{\nu}_{\mu} \rightarrow \bar{\nu}_{e}}=A_{\mu ; e} \sin ^{2} \frac{\Delta m_{41}^{2} L}{4 E},
$$

where $\Delta m_{41}^{2} \equiv \Delta m_{\mathrm{LSND}}^{2}, L$ is the distance between source and detector and $E$ is the neutrino energy. We use the abbreviation $\Delta m_{k j}^{2} \equiv m_{k}^{2}-m_{j}^{2}$. The oscillation amplitude $A_{\mu ; e}$ is given by

$$
A_{\mu ; e}=4\left(1-c_{e}\right)\left(1-c_{\mu}\right)
$$

with

$$
c_{\alpha}=\sum_{j=1}^{3}\left|U_{\alpha j}\right|^{2} \quad(\alpha=e, \mu) .
$$

From the results of reactor and accelerator disappearance experiments it follows that [14]

$$
c_{\alpha} \leq a_{\alpha}^{0} \quad \text { or } \quad c_{\alpha} \geq 1-a_{\alpha}^{0}
$$

with $a_{\alpha}^{0}=\frac{1}{2}\left(1-\sqrt{1-B_{\alpha ; \alpha}^{0}}\right)$, where $B_{\alpha ; \alpha}^{0}$ is the upper bound for the amplitude of $\nu_{\alpha} \rightarrow \nu_{\alpha}$ oscillations. The exclusion plots obtained from the Bugey [18] and CDHS [19] and CCFR [20] experiments imply that $a_{e}^{0} \lesssim 4 \times 10^{-2}$ for $\Delta m_{\text {LSND }}^{2} \gtrsim 0.1 \mathrm{eV}^{2}$ and $a_{\mu}^{0} \lesssim 0.2$ for $\Delta m_{\text {LSND }}^{2} \gtrsim 0.4 \mathrm{eV}^{2}$ [21. Below $\Delta m^{2} \simeq 0.3 \mathrm{eV}^{2}$, the survival amplitude $B_{\mu ; \mu}$ is not restricted by experimental data, i.e., $B_{\mu ; \mu}^{0}=1$.
The survival probability of solar $\nu_{e}$ 's is bounded by $P_{\nu_{e} \rightarrow \nu_{e}}^{\odot} \geq\left(1-c_{e}\right)^{2}$ [14. Therefore, to be in agreement with the results of solar neutrino experiments we conclude that from the two ranges of $c_{e}$ in Eq.(5) only

$$
c_{e} \geq 1-a_{e}^{0}
$$

is allowed.

We will address now the question of what information on the parameter $c_{\mu}$ can be obtained from the asymmetry $A_{\mu}$ (11). As a first step we derive an upper bound on the number of downward-going $\mu$-like events $D$. The probability of $\nu_{\alpha} \rightarrow \nu_{\alpha}$ and $\bar{\nu}_{\alpha} \rightarrow \bar{\nu}_{\alpha}$ transitions of atmospheric neutrinos is given by

$$
\begin{aligned}
& P_{\nu_{\alpha} \rightarrow \nu_{\alpha}}=P_{\bar{\nu}_{\alpha} \rightarrow \bar{\nu}_{\alpha}}= \\
& \left.\left|\sum_{j=1,2}\right| U_{\alpha j}\right|^{2}+\left.\left|U_{\alpha 3}\right|^{2} \exp \left(-i \frac{\Delta m_{31}^{2} L}{2 E}\right)\right|^{2}+\left|U_{\alpha 4}\right|^{4},
\end{aligned}
$$

where we have taken into account that $\Delta m_{41}^{2} \gg \Delta m_{31}^{2}$ and $\Delta m_{21}^{2} L / 2 E \ll 1\left(\Delta m_{21}^{2}\right.$ is relevant for solar neutrinos). Because of the small value of $\Delta m_{\mathrm{atm}}^{2} \equiv \Delta m_{31}^{2}$, it is well fulfilled that downward-going neutrinos do not oscillate with the atmospheric mass-squared difference.f Therefore, we obtain for the survival probability of downward-going neutrinos

$$
P_{\nu_{\alpha} \rightarrow \nu_{\alpha}}^{D}=c_{\alpha}^{2}+\left(1-c_{\alpha}\right)^{2} .
$$

Furthermore, conservation of probability and Eq.(6) allow to deduce the upper bound

$$
P_{\nu_{e} \rightarrow \nu_{\mu}}^{D} \leq 1-P_{\nu_{e} \rightarrow \nu_{e}}^{D}=2 c_{e}\left(1-c_{e}\right) \leq 2 a_{e}^{0}\left(1-a_{e}^{0}\right) .
$$

Note that all arguments hold for neutrinos and antineutrinos. Denoting the number of muon (electron) neutrinos and antineutrinos produced in the atmosphere by $n_{\mu}$ $\left(n_{e}\right)$, from Eqs.(8) and (9) we have the upper bound

$$
D \leq n_{\mu}\left[c_{\mu}^{2}+\left(1-c_{\mu}\right)^{2}\right]+2 n_{e} a_{e}^{0}\left(1-a_{e}^{0}\right) .
$$

Taking into account only the part of $D$ which is determined by the $\stackrel{(-)}{\nu}$ ) survival probability, we immediately obtain the lower bound

$$
D \geq n_{\mu}\left[c_{\mu}^{2}+\left(1-c_{\mu}\right)^{2}\right] .
$$

Considering only $\left|U_{\mu 4}\right|^{4}$ in Eq.(7), we readily arrive at a lower bound on $U$ as well:

${ }^{*}$ This is not completely true for neutrino directions close to the horizon with $\Delta m_{\mathrm{atm}}^{2} \gtrsim 3 \times 10^{-3} \mathrm{eV}^{2}$. Taking into account the result of the CHOOZ experiment [22], we have checked, however, that numerically this has a negligible impact on the following discussion. 


$$
U \geq n_{\mu}\left(1-c_{\mu}\right)^{2}
$$

This inequality is analogous to the above inequality for the survival of solar neutrinos and is valid also with matter effects in the earth.

Now we can assemble the inequalities (10), (11) and (12) and it follows the main result of this work

$$
-A_{\mu} \leq \frac{c_{\mu}^{2}+2 a_{e}^{0}\left(1-a_{e}^{0}\right) / r}{c_{\mu}^{2}+2\left(1-c_{\mu}\right)^{2}},
$$

where we have defined $r \equiv n_{\mu} / n_{e}$. For the numerical evaluation of Eq.13 we use $-A_{\mu} \geq 0.254$ at $90 \%$ CL, the $90 \%$ CL bound $a_{e}^{0}$ from the result of the Bugey experiment and $r=2.8$ read off from Fig. 3 in Ref. [1] of the Super-Kamiokande Collaboration. As a result we get

$$
c_{\mu} \geq a_{\mathrm{SK}} \simeq 0.45,
$$

as can be seen from the horizontal line in Fig. 2. Note that the dependence of this lower bound on $\Delta m_{\mathrm{LSND}}^{2} \equiv$ $\Delta m_{41}^{2}$ is almost negligible due to the smallness of the second term in the numerator on the right-hand side of Eq.(13). Consequently, also the exact value of $r$ is not important numerically.

In Fig. 2 2 we have also depicted the bounds

$$
c_{\mu} \leq a_{\mu}^{0} \quad \text { and } \quad c_{\mu} \geq 1-a_{\mu}^{0}
$$

that were obtained from the exclusion plot of the CDHS $\nu_{\mu}$ disappearance experiment. For $\Delta m_{\mathrm{LSND}}^{2} \simeq 0.24 \mathrm{eV}^{2}$ these two bounds meet at $c_{\mu}=0.5$. Below $0.24 \mathrm{eV}^{2}$ there are no restrictions on $c_{\mu}$ from SBL experiments.

Finally, we take into account the result of the LSND experiment, from which information on the SBL $\bar{\nu}_{\mu} \rightarrow \bar{\nu}_{e}$ transition amplitude $A_{\mu ; e}$ (3) is obtained. Using Eq.(6) and the lower bound $A_{\mu ; e}^{\min }$, which can be inferred from the region allowed by LSND, we derive the further bound on $c_{\mu}$ [23]

$$
c_{\mu} \leq a_{\mathrm{LSND}} \equiv 1-A_{\mu ; e}^{\min } / 4 a_{e}^{0} .
$$

This bound is represented by the curve in Fig. 2 labelled LSND + Bugey.

Fig. 2 clearly shows that a four-neutrino mass hierarchy is strongly disfavored because no allowed region for $c_{\mu}$ is left in this plot. A four-neutrino mass hierarchy is also strongly disfavored for $\Delta m_{\text {LSND }}^{2} \gtrsim 0.4 \mathrm{eV}^{2}$ as was shown in Ref. 14. We want to stress that all bounds are derived from $90 \%$ CL plots and that the bound (16) is quite sensitive to the actual values of $A_{\mu ; e}^{\min }$ and $a_{e}^{0}$. This has to be kept in mind in judging the result derived here. As was noticed before [14], the procedure discussed here applies to all four-neutrino mass spectra of class 1 where a group of three neutrino masses is close together and separated from the fourth neutrino mass by a gap needed to explain the result of the LSND experiment. The reason is that all arguments presented here remain unchanged if one defines $c_{\alpha}$ (3) by a summation over the indices of the three close masses for each of the mass spectra of class 1 (see Fig. 1), i.e., $j=1,2,3$ for the spectra I and II and $j=2,3,4$ for the spectra III and IV.

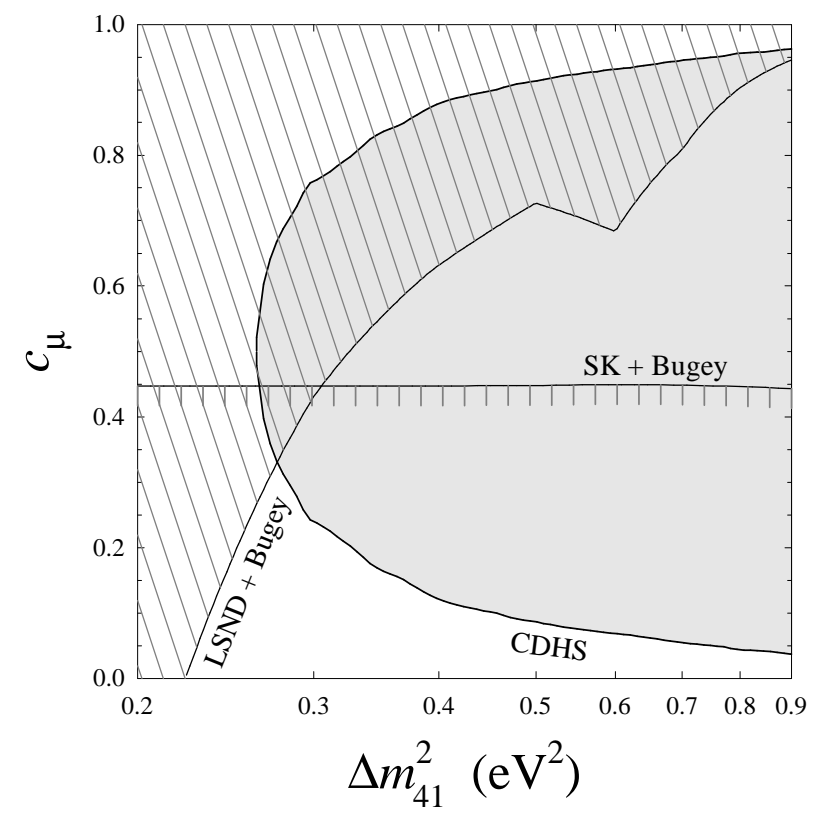

FIG. 2. Regions in the $\Delta m_{41}^{2}-c_{\mu}$ plane disfavored by the results of the CDHS, LSND, Super-Kamiokande and Bugey experiments in the case of the spectra of class 1 . The shaded region is excluded by the inequalities (15) and the hatched region by the bound 16 . The nearly horizontal curve labelled SK + Bugey represents the lower bound (13) derived from the Super-Kamiokande up-down asymmetry. Since this bound lies above the white region allowed by inequalities (15) and (16), the spectra of class 1 are disfavored by the data.

To give an intuitive understanding that the data disfavor all spectra of class 1 we note that $c_{\mu}$ cannot be too close to 1 in order to explain the non-zero LSND $\bar{\nu}_{\mu} \rightarrow \bar{\nu}_{e}$ oscillation amplitude (3). On the other hand, if $c_{\mu}$ is too close to zero, the atmospheric $\nu_{\mu}$ oscillations are suppressed (see Eq.(7), taking into account that $\left.\left|U_{\mu 4}\right|^{2}=1-c_{\mu}\right)$. For $\Delta m_{\mathrm{LSND}}^{2} \lesssim 0.3 \mathrm{eV}^{2}$ these two requirements contradict each other. For $\Delta m_{\mathrm{LSND}}^{2} \gtrsim 0.3 \mathrm{eV}^{2}$ they are in contradiction to the results of the CDHS and CCFR $\nu_{\mu}$ disappearance experiments requiring $c_{\mu}$ to be either close to zero or 1 (see Eq.(50)).

According to the previous discussion, only the mass spectra of class 2 remain. They can be characterized in the following way:

$$
\underbrace{\overbrace{m_{1}<m_{2}}^{\text {atm }} \ll \overbrace{m_{3}<m_{4}}^{\text {solar }}}_{\text {LSND }}
$$

and

$$
\underbrace{\overbrace{m_{1}<m_{2}}^{\text {solar }} \ll \overbrace{m_{3}<m_{4}}^{\text {atm }}}_{\text {LSND }} .
$$


Let us now discuss which impact the up-down asymmetry $A_{\mu}$ has on these mass schemes. We consider first scheme (A) and go through the same steps as in the case of the mass hierarchy. Now we define

$$
c_{\alpha}=\sum_{j=1,2}\left|U_{\alpha j}\right|^{2} .
$$

Then the results of reactor experiments and the energydependent suppression of the solar neutrino flux leads to

$$
c_{e} \leq a_{e}^{0} .
$$

Repeating the derivation of Eq.(13) with $c_{\alpha}$ as defined in Eq.(19), it is easily seen that the inequality (13) holds also for scheme (A). On the other hand, the bound that takes into account the LSND result now has the form

$$
c_{\mu} \geq A_{\mu ; e}^{\min } / 4 a_{e}^{0} .
$$

The corresponding curve in the $\Delta m_{41}^{2}-c_{\mu}$ plane is given by a reflection of the curve labelled LSND + Bugey in Fig. 2 at the horizontal line $c_{\mu}=0.5$. Therefore, in the case of scheme (A) the allowed region of $c_{\mu}$ is determined by the bound (21) and by $c_{\mu} \geq 1-a_{\mu}^{0}$. This region is allowed and not restricted by $c_{\mu} \gtrsim 0.45$ obtained from the Super-Kamiokande up-down asymmetry.

A discussion of scheme (B) with $c_{e} \geq 1-a_{e}^{0}$ leads to the bound (13) with $c_{\mu}$ replaced by $1-c_{\mu}$ in this formula and to Eq.(16). Therefore, the bounds for scheme (B) are obtained from those of scheme (A) by a reflection of the curves at the line $c_{\mu}=0.5$. In summary, the white area in Fig. 2 represents the allowed region for $1-c_{\mu}$ in scheme (A) and for $c_{\mu}$ in scheme (B).

In this paper we have shown that the existing neutrino oscillation data allow to draw definite conclusions about the nature of the possible four-neutrino mass spectra. We have demonstrated that the spectra (I) - (IV) in Fig. 1, including the hierarchical one, are all disfavored by the data in the whole range $0.2 \mathrm{eV}^{2} \lesssim \Delta m_{\mathrm{LSND}}^{2} \lesssim 2 \mathrm{eV}^{2}$ of the mass-squared difference determined by LSND and other SBL neutrino oscillation experiments. With the Super-Kamiokande result on the atmospheric up-down asymmetry it has been also possible to investigate the region $\Delta m_{\mathrm{LSND}}^{2} \lesssim 0.3 \mathrm{eV}^{2}$ which was not explored in previous publications. The only four-neutrino mass spectra that can accommodate all the existing neutrino oscillation data are the spectra (A) and (B) in Fig. 11 in which two pairs of close masses are separated by the LSND mass gap. The analysis introduced in this paper enables us in addition to obtain information on the mixing matrix $U$ via a rather stringent bound on the quantity $c_{\mu}(19)$ for the allowed schemes (A) and (B).

\section{ACKNOWLEDGMENTS}

S.M.B. would like to thank the Institute for Theoretical Physics of the University of Vienna for its hospitality.
[1] Y. Fukuda et al., Super-Kamiokande Coll., Phys. Rev. Lett. 81, 1562 (1998).

[2] Y. Fukuda et al., Kamiokande Coll., Phys. Lett. B 335, 237 (1994).

[3] R. Becker-Szendy et al., IMB Coll., Nucl. Phys. B (Proc. Suppl.) 38, 331 (1995).

[4] W.W.M. Allison et al., Soudan Coll., Phys. Lett. B 391 , 491 (1997); preprint hep-ex/9901024 (1998).

[5] M. Ambrosio et al., MACRO Coll., Phys. Lett. B 434, 451 (1998).

[6] B.T. Cleveland et al., Astrophys. J. 496, 505 (1998).

[7] K.S. Hirata et al., Kamiokande Coll., Phys. Rev. Lett. 77, 1683 (1996).

[8] W. Hampel et al., GALLEX Coll., Phys. Lett. B 388, 384 (1996).

[9] D.N. Abdurashitov et al., SAGE Coll., Phys. Rev. Lett. 77, 4708 (1996).

[10] Y. Fukuda et al., Super-Kamiokande Coll., Phys. Rev. Lett. 81, 1158 (1998).

[11] C. Athanassopoulos et al., LSND Coll., Phys. Rev. Lett. 77, 3082 (1996); Phys. Rev. Lett. 81, 1774 (1998); LSND WWW page: http://www.neutrino.lanl.gov/LSND/.

[12] J.T. Peltoniemi, D. Tommasini and J.W.F. Valle, Phys. Lett. B 298, 383 (1993); J.T. Peltoniemi and J.W.F. Valle, Nucl. Phys. B 406, 409 (1993); D.O. Caldwell and R.N. Mohapatra, Phys. Rev. D 48, 3259 (1993); E. Ma and P. Roy, ibid 52, R4780 (1995).

[13] S.M. Bilenky, C. Giunti and W. Grimus, hep-ph/9812360 (1998).

[14] Proc. of Neutrino '96, Helsinki, June 1996, edited by K. Enqvist et al., p. 174, World Scientific, Singapore, 1997 hep-ph/9609343); S.M. Bilenky, C. Giunti and W. Grimus, Eur. Phys. J. C 1, 247 (1998).

[15] N. Okada and O. Yasuda, Int. J. Mod. Phys. A 12, 3669 (1997).

[16] V. Barger, S. Pakvasa, T.J. Weiler and K. Whisnant, Phys. Rev. D 58, 093016 (1998).

[17] Talk by K. Scholberg at Neutrino Telescopes, Venice, February $23-26,1999$.

[18] B. Achkar et al., Bugey Coll., Nucl. Phys. B 434, 503 (1995).

[19] F. Dydak et al., CDHS Coll., Phys. Lett. B 134, 281 (1984).

[20] I.E. Stockdale et al., CCFR Coll., Phys. Rev. Lett. 52, 1384 (1984).

[21] S.M. Bilenky, A. Bottino, C. Giunti and C.W. Kim, Phys. Rev. D 54, 1881 (1996).

[22] M. Apollonio et al., CHOOZ Coll., Phys. Lett. B 420, 397 (1998).

[23] S.M. Bilenky, C. Giunti and W. Grimus, Proc. of the $5^{\text {th }}$ Workshop on High Energy Physics Phenomenology, IUCAA, Pune, India, January, 12 - 26, PRAMANA 51, 51 (1998). 